\title{
Solid phase extraction as an innovative separation method for measuring free and entrapped drug in lipid nanoparticles
}

\author{
Alexis Guillot ${ }^{1,2,},{ }^{,}$, , Anne-Claude Couffin ${ }^{3,4}$, Xavier Sejean ${ }^{3,4}$, Fabrice Navarro ${ }^{3,4}$ \\ Markus Limberger ${ }^{1, \ddagger}$ and Claus-Michael Lehr ${ }^{2,5}$ \\ 1 PHAST GmbH, Kardinal-Wendel-Str. 16, D-66424 Homburg, Germany \\ 2 Helmholtz Institute for Pharmaceutical Research Saarland (HIPS), Helmholtz Center for \\ Infection Research (HZI), Department DDEL, Saarland University, Campus A41, D-66123 \\ Saarbrücken, Germany. \\ 3 Univ Grenoble Alpes, F-38000 Grenoble, France. \\ 4 CEA - LETI Minatec, Technologies for Healthcare and Biology Division, 17, rue des Martyrs, \\ F-38054 Grenoble Cedex 09, France.
}

5 Saarland University, Department of Pharmacy, Campus A41, D-66123 Saarbrücken, Germany

* Corresponding author, e-mail: alexis.guillot@phast.com

† Current address: PHAST Development GmbH \& Co. KG, Byk-Gulden-Str. 2, Campus Konstanz,

D-78467 Konstanz, Germany

\# Current address: Joseph-Haydn-Str. 41, D-66125 Saarbrücken, Germany 


\section{ABSTRACT}

\section{Purpose}

Contrary to physical characterization techniques for nanopharmaceuticals (shape, size and zeta-potential), the techniques to quantify the free and the entrapped drug remain very few and difficult to transpose in routine analytical laboratories. The application of Solid Phase Extraction (SPE) technique was investigated to overcome this challenge.

\section{Methods}

The separation of free and entrapped drug by SPE was quantitatively validated by High Performance Liquid Chromatography. The developed protocol was implemented to characterize cyclosporine A-loaded $120 \mathrm{~nm}$-sized lipid nanoparticles (LNPs, Lipidot ${ }^{\circledR}$ ) dispersed in aqueous buffer. The colloidal stability was assessed by Dynamic Light Scattering (DLS).

\section{Results}

Validation experiments demonstrated suitable linearity, repeatability, accuracy and specificity to quantify residual free, entrapped and total drug. For the investigated LNPs, the method revealed a very limited shelflife of the formulation when stored in an aqueous buffer at $5{ }^{\circ} \mathrm{C}$ and even more at elevated temperature. Nevertheless, the DLS measurements confirmed the stability of nanoparticles during SPE in a suitable concentration range.

\section{Conclusions}

SPE, when successfully validated, represents a valuable tool for drug development and quality control purposes of lipid-based nanopharmaceuticals in an industrial environment.

KEYWORDS: Solid-phase extraction; Lipid nanoemulsion; Nanoparticles; Cyclosporine; Separation techniques 


\section{LIST OF ABBREVIATIONS}

ACN Acetonitrile

AP

Active pharmaceutical ingredient

CSA

Cyclosporine A

CV

Coefficient of Variation

DAD

Diode Array Detector

DLS

Dynamic Light Scattering

EDCD

Entrapped Drug Content Determination

F0

Fraction 0

F1

Fraction 1

F2

Fraction 2

FDCD

Free Drug Content Determination

HPLC

High Performance Liquid Chromatographie

LNP

Lipid Nanoparticle

$\mathrm{ICH}$

International Conference on Harmonisation of Technical Requirements

for Registration of Pharmaceuticals for Human Use

LOD Limit of Detection

LOQ Limit of Quantification

$\mathrm{MeOH} \quad$ Methanol

MWCO Molecular Weight Cut-Off

n. a. Not available/not applicable

NP Nanoparticle

PBS

Phosphate Buffered Saline 
Polydispersity Index

PEG

Polyethylene Glycol

$\mathrm{R}^{2}$

Determination coefficient

$\mathrm{RP}$

Reverse Phase

rpm

Revolutions per minute

RSD

Relative Standard Deviation

SPE

Solid Phase Extraction

TDCD

Total Drug Content Determination

TFA

Trifluoroacetic acid $0.5 \%(\mathrm{v} / \mathrm{v})$ 


\section{INTRODUCTION}

In the scientific literature, nanomedicines are often considered as a promising field that could offer many advantages over small molecules. Many nanoproducts are currently under clinical trial or have already been approved, such as Abraxane ${ }^{\circledR}$ (paclitaxel) Ferumoxytol ${ }^{\circledR}$ (iron supplement) or Amphotec ${ }^{\circledR}$ (amphotericin B) for instance. However, the path until the final status of "approved" appears to be harder as hoped(1). The complexity of the nanotechnologies for human health is such that time consuming missteps are unfortunately very common(2). In particular, suitable techniques for the physicochemical characterization of the product are of the highest importance to reliably interpret results of the next studies (in vivo tests, toxicity, etc.). The shape, the size or the zeta-potential of nanoparticles are key parameters for which many techniques(3) are already available and thoroughly investigated. On the contrary, far fewer techniques can be implemented in routine to investigate the distribution of the Active Pharmaceutical Ingredients (API) within the different compartments of the nanoformulation, essentially to distinguish between the free and entrapped drug. Dialysis methods are common for this purpose but the interpretation of the results is often not so obvious(4). Other separation techniques like Cross-Flow-Filtration, Field-Flow Fractionation, Ultracentrifugation, Capillary Electrophoresis, Size Exclusion Chromatography or Hydrodynamic Chromatography have been proposed in the scientific literature(5). Yet, simple and reliable methods, as required for quality control purposes, may not be generally feasible for any nanosystem. In this aim, pressure ultrafiltration was already proposed as a suitable separation technique for in vitro drug release tests(6). Further investigations revealed that pressure ultrafiltration for liposomes could instantaneously completely achieve the separation whereas other techniques failed(7).

For the present study, the SPE technique was selected because routine analysis can easily be performed at a later stage, as it is the case for pressure ultrafiltration. It generally allows the full automation of the analytical procedure and hence, a strong reproducibility. Moreover, SPE has already been successfully applied for liposomes $(8,9)$, suggesting that it may be suitable for lipid nanoparticles (LNP) as well. This paper deals with the development of SPE 
methods to quantify separately free and entrapped cyclosporine A in a patented nanocarrier: Lipidot $^{\circledR}(10)$. The colloidal stability of the $120 \mathrm{~nm}$-sized LNPs passed through SPE-cartridges was verified by monitoring the particle size. The subsequent quantification of the API was performed by High Performance Liquid Chromatography (HPLC). The developed protocol as presented in Figure 1 consisted of a tripartite SPE-method including a Total Drug Content Determination method (TDCD), an Entrapped Drug Content Determination method (EDCD) and a Free Drug Content Determination method (FDCD).

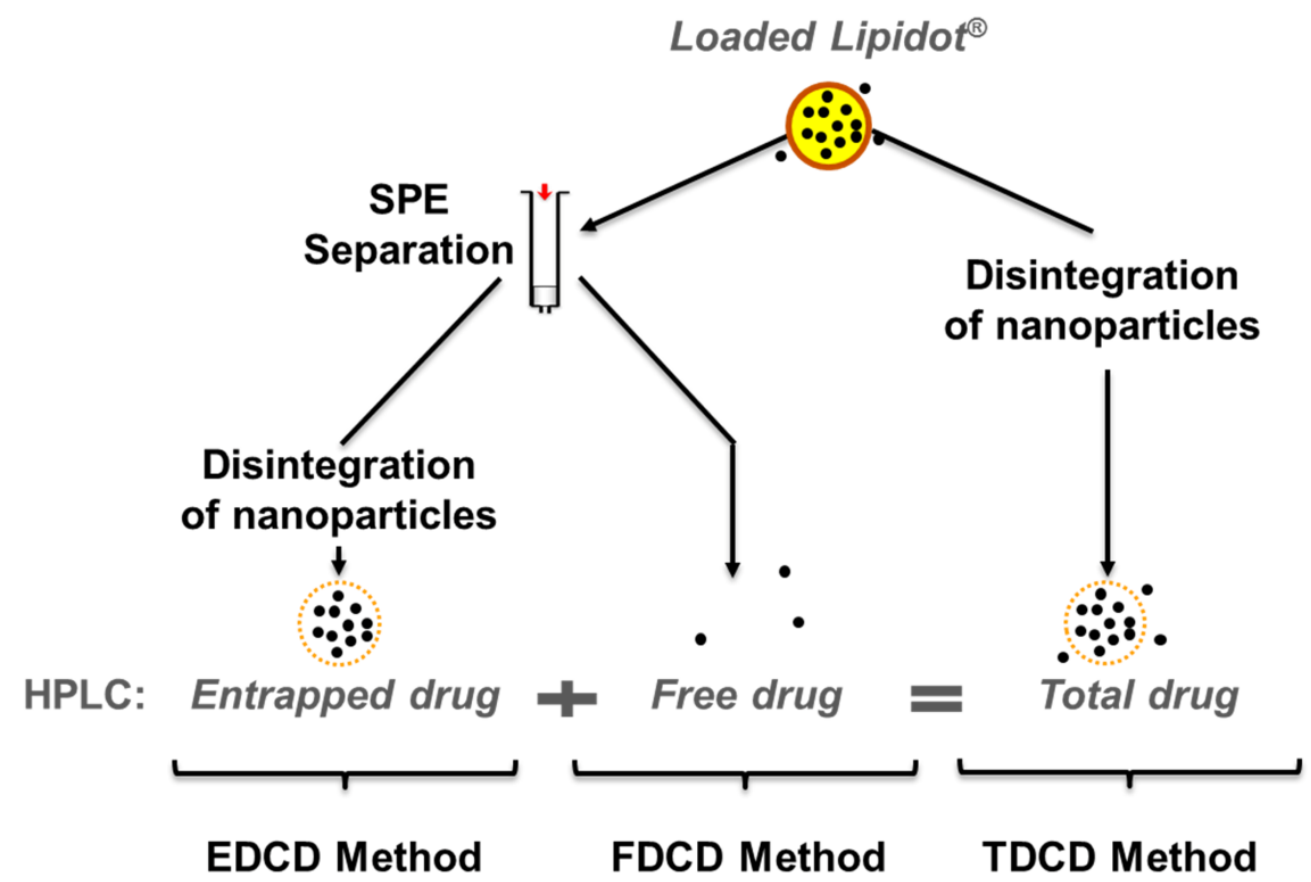

Figure 1: $\quad$ Simplified process description of the tripartite SPE-method

After validation of the analytical procedure according to the $\mathrm{ICH}$ guideline $\mathrm{Q} 2 \mathrm{~A}(11)$, the protocol was implemented for the Lipidot ${ }^{\circledR}$ formulation. Finally, a $48 \mathrm{~h}$-stress test at elevated temperature and stirring speed was performed to evaluate the stability of the nanoformulation. 


\section{MATERIALS AND METHODS}

\subsection{Materials}

Suppocire NB $^{\text {TM }}$ was purchased from Gattefosse S.A. (Saint-Priest, France). Myrj ${ }^{\mathrm{TM}} \mathrm{S} 40$ (PEG 40 stearate, 1,980 Da) and Super Refined Soybean Oil were obtained from Croda Uniquema (Chocques, France). Lipoid ${ }^{\circledR} S 75$ (soybean lecithin at $69 \%$ of phosphatidylcholine) provided by Lipoid $\mathrm{GmbH}$ (Ludwigshafen, Germany). All these excipients are pharmaceutical grade and used as received. Acetonitrile (ACN) and methanol (MeOH) were provided by LiChrosolv ${ }^{\circledR}$, orthophosphoric acid $85 \%$ by BDH Prolabo ${ }^{\circledR}$ and trifluoroacetic acid 99.9 \% by EMD Millipore Corporation. All reagents were HPLC-grade. The cyclosporine A (CSA) was supplied by RTC Pharma. Highly purified water was produced using a Milli- $Q^{\circledR}$ Gradient $A 10$ from EMD Millipore Corporation. Lipidots are formulated using a VCX750 Ultrasonic processor from Sonics (Newtown, USA) equipped with a $3 \mathrm{~mm}$ diameter microtip. The balance was an AX205 from Mettler Toledo, the water bath was the type 19 of Julabo, the centrifuge was a Minispin from Eppendorf and the vortex was a Vortex-Genie 2 from Scientific Industries. The quantifications of the active pharmaceutical ingredient (API) were performed by high performance liquid chromatography (HPLC) using an Agilent 1100 Series system from Agilent Technologies with a degasser, oven and DADdetector. The HPLC-column was an XTerra ${ }^{\circledR}$ RP-18 $(5 \mu \mathrm{m} \times 150 \mathrm{~mm} \times 4.6 \mathrm{~mm})$ from Waters. The separation of the drug from nanoparticles was carried out using a SPE system from Supelco: Visiprep ${ }^{\mathrm{TM}}$ 12-Port Vacuum Manifolds. The SPE-cartridges Supelclean ${ }^{\mathrm{TM}}$ LC-18 SPE tube ( $1 \mathrm{~mL}, 60 \AA$ pore size, $45 \mu \mathrm{m}$ particle size) were provided by Supelco. The nanoparticle size experiments were performed using a Zetasizer Nano ZS from Malvern Instrument equipped with a $532 \mathrm{~nm}$ green laser source. The stress test was carried out using the Manual Diffusion Test System equipped with a $7 \mathrm{~mL}$ vertical diffusion cell provided by Hanson Research. 


\section{$2.2 \quad$ Lipidot $^{\circledR}$ formulation}

The formulation of conventional Lipidot ${ }^{\circledR}$ has been previously described elsewhere $(12,13)$. The lipid phase was prepared by mixing lipophilic ingredients: wax, oil and Lipoid S75 phospholipids, whereas the aqueous phase was composed of the hydrophilic PEG surfactant, MyrjS40, solubilized in 1X PBS aqueous buffer. After melting the oil phase and homogeneous mixing with the aqueous phase at $45^{\circ} \mathrm{C}$, both phases were crudely mixed and sonication cycles are performed during 20 min with intervals of $10 \mathrm{~s}$ "Pulse On" and $30 \mathrm{~s}$ "Pulse Off".. The purification step was carried out overnight using dialysis (1X PBS, MWCO: $12 \mathrm{kDa}$, regenerated cellulose membrane, Spectra/Por ${ }^{\circledR}$ ). Lipid nanoparticles (LNP) were finally formulated at a theoretical total concentration of lipids of $60 \mathrm{mg} / \mathrm{mL}$ and then filtered through a $0.22 \mu \mathrm{m}$ cellulose Millipore membrane. Typically, for $120 \mathrm{~nm}$-sized formulation, particles are composed of the $43 \%(\mathrm{w} / \mathrm{w})$ of dispersed phase (860 mg of total excipients for $2 \mathrm{~mL}$-volum of formulation) with a surfactant/core ratio of 0.43 and a lecithin/PEG surfactant weight ratio of 0.21 . Concerning the manufacturing of the CSA-loaded LNPs, an appropriate amount of CSA in absolute ethanol ( $45 \mu \mathrm{L}$ of $63.7 \mathrm{mg} / \mathrm{mL}$ of CSA stock solution) was initially added to the oily phase (local concentration of CSA in oily phase: $0.48 \% \mathrm{w} / \mathrm{w}$ ), and then the solvent was evaporated under argon flow. The nanoparticles encapsulating CSA were then formulated as above described. The purification step by dialysis was extended until $72 \mathrm{~h}$ to leave time non-entrapped CSA to remove itself due to its low solubility in aqueous buffer. CSA drug was initially loaded until a concentration of $210 \mu \mathrm{g} / \mathrm{mL}$ and a total lipid concentration of $60 \mathrm{mg} / \mathrm{mL}$. The blank nanoparticles were prepared without drug corresponded to the placebo formulation. 
The solution of trifluoroacetic acid $0.5 \%(\mathrm{v} / \mathrm{v})$ (hereafter named TFA), was prepared diluting trifluoroacetic acid $99.9 \%$ with highly purified water. The solution of $\mathrm{ACN}: \mathrm{H}_{3} \mathrm{PO}_{4}$ $5 \mathrm{mM}(75: 25 ; \mathrm{v} / \mathrm{v})$ and the solution of $\mathrm{MeOH}: \mathrm{H}_{3} \mathrm{PO}_{4} 5 \mathrm{mM}(75: 25 ; \mathrm{v} / \mathrm{v})$ were prepared mixing phosphoric acid $5 \mathrm{mM}$ solution with respectively $\mathrm{ACN}$ and $\mathrm{MeOH}$. The solution of phosphoric acid $5 \mathrm{mM}$ was prepared by dilution of orthophosphoric acid $85 \%$ with highly purified water.

\subsection{Principle of the tripartite SPE-method for lipid nanoparticles}

The samples formerly stored at $5{ }^{\circ} \mathrm{C}$ were left without handling until equilibration at room temperature. As described in Table 1, media were successively introduced into the SPEcartridges for cleaning and equilibration prior to introduction of the samples of formulation. The nanoparticles were eluted in a first fraction (F1) adding TFA as eluent into the SPEcartridge. The free drug was eluted in a second fraction (F2) using ACN as a second eluent. The pressure in the vacuum chamber during the elution of LNPs (Step 3) was adjusted, resulting in a flow rate of $1 \mathrm{~mL} / \mathrm{min}$.

The nanoparticles eluted in F1 could further be disintegrated to release entrapped drug. Briefly, the disintegration step consisted in mixing one volume of eluted nanoparticles with two volumes of acetonitrile. The mixture was vortexed and centrifuged at 10,000 rpm for 10 min. The released drug in supernatant was quantified using a suitable HPLC method (EDCD method).

The free drug eluted in F2 could be directly quantified by HPLC (FDCD method).

The total drug content was determined for a sample without being passed through SPE but directly prepared as previously described in the disintegration step. The released entrapped drug and the free drug were quantified by HPLC (TDCD method). 
Table 1: SPE overall plan.

\begin{tabular}{|c|c|c|c|c|c|c|}
\hline Step & Medium & Volume & Pressure & Fraction & Container & Function \\
\hline 1 & $\mathrm{ACN}$ & $\approx 6 \mathrm{~mL}$ & $\approx-50 \mathrm{kPa}$ & \multirow{2}{*}{ Fo } & \multirow{2}{*}{ Waste } & Cleaning \\
\hline 2 & TFA & $\approx 4 \mathrm{~mL}$ & $\approx-50 \mathrm{kPa}$ & & & Equilibration \\
\hline 3 & Sample & $1 \mathrm{~mL}$ & $\approx-20 \mathrm{kPa}$ & \multirow{2}{*}{$\mathrm{F} 1$} & \multirow{2}{*}{$5 \mathrm{~mL}-\mathrm{VF} 1$} & \multirow{2}{*}{ Elution of NPs } \\
\hline 4 & TFA & $\approx 3 \mathrm{~mL}$ & $\approx-50 \mathrm{kPa}$ & & & \\
\hline 5 & $\mathrm{ACN}$ & $\approx 4 \mathrm{~mL}$ & $\approx-50 \mathrm{kPa}$ & $\mathrm{F} 2$ & 5 mL-VF2 & Elution of API \\
\hline \multicolumn{7}{|c|}{ ACN: Acetonitrile; TFA: Trifluoroacetic acid $0.5 \%$ (v/v); Sample: Nanoformulation to be tested; NPs: nanoparticles; API: Active Pharmaceutica } \\
\hline
\end{tabular}

\subsection{Assessment of the colloidal stability of Lipidot ${ }^{\circledR}$ following SPE protocol}

Size distribution of Lipidot ${ }^{\circledR}$ was investigated by DLS to monitor the colloidal integrity of the nanoparticles after being applied SPE method. Prior to SPE, the sample of placebo Lipidot ${ }^{\circledR}$ was diluted using TFA until 3, 6, 10, 15, 30 and $60 \mathrm{mg} / \mathrm{mL}$ LNPs. Each dilution of the set was further eluted by SPE as described in Table 1 until step 4. The fractions F1 were collected and the particle sizes were assessed using the Zetasizer Nano ZS. The solution of TFA was used as blank and placebo Lipidot ${ }^{\circledR}$ as negative control. Before measurement, samples were diluted in TFA to a dispersed phase weight fraction of $1 \mathrm{mg} / \mathrm{mL}$ in order to avoid multiple scattering effects. All samples were prepared in duplicate and analyzed in triplicate. The Zaverage diameter (Size, $\mathrm{nm}$ ) and polydispersity index (Pdl) of the lipid nanoparticles were extracted from the second cumulant of the correlation function of the intensity distribution. Each result was the mean of three independent measurements performed at $25^{\circ} \mathrm{C}$, at a fixed angle of $173^{\circ}$.

\subsection{SPE separation methods and validation by HPLC analysis}

The quantitative validation of the SPE method for the FDCD was performed for a set of placebo Lipidot ${ }^{\circledast}$ diluted with highly purified water solutions spiked with different proportions of cyclosporine A. A CSA stock solution was prepared in duplicate dissolving the powder of 
CSA in acetonitrile until a concentration of $2842 \mu \mathrm{g} / \mathrm{mL}$. A first CSA dilution set was then prepared by dilution of the stock solution using ACN until 284, 426, 710, 852, 1136, 1421 and $1705 \mu \mathrm{g} / \mathrm{mL}$ CSA. The dilution set was further diluted with a dilution factor of 50 using highly purified water, so that the resulting dilution set contained $2 \%,(v / v)$ acetonitrile, i.e. less than the limit of $5 \%$ above which LNPs disintegrate (unpublished DLS results). The sample of placebo Lipidot ${ }^{\circledR}$ was added until a concentration of $9.6 \mathrm{mg} / \mathrm{mL}$, i.e. until a LNP dilution factor of 6.25 (dilution set designated by "CSA + placebo Lipidot ${ }^{\circledR}$ for FDCD" in Table 2). In addition, another dilution set ("CSA for FDCD" in Table 2) was prepared without placebo Lipidot $^{\circledR}$. Each sample of both the dilution sets of CSA solutions and CSA-spiked placebo LNPs was investigated in triplicate by solid phase extraction according to the validation plan presented in Figure 2 and using the SPE protocol previously described in Table 1.

Table 2: Dilution sets prepared for the validation of the tripartite-SPE method.

\begin{tabular}{|c|c|c|c|c|c|c|c|c|}
\hline & \multicolumn{2}{|c|}{ CsA for FDCD } & \multicolumn{2}{|c|}{$\begin{array}{c}\text { CSA + placebo } \\
\text { Lipidot }^{\circledR} \text { for FDCD }\end{array}$} & \multicolumn{2}{|c|}{$\begin{array}{c}\text { CSA + placebo } \\
\text { Lipidot }^{\circledR} \text { for EDCD }\end{array}$} & \multicolumn{2}{|c|}{$\begin{array}{c}\text { CSA + placebo } \\
\text { Lipidot }^{\circledR} \text { for TDCD }\end{array}$} \\
\hline & $\begin{array}{c}\text { CSA } \\
(\mu \mathrm{g} / \mathrm{mL})\end{array}$ & $\begin{array}{c}\text { LNPs } \\
(\mathrm{mg} / \mathrm{mL})\end{array}$ & $\begin{array}{c}\text { CSA } \\
(\mu \mathrm{g} / \mathrm{mL})\end{array}$ & $\begin{array}{c}\text { LNPs } \\
(\mathrm{mg} / \mathrm{mL})\end{array}$ & $\begin{array}{c}\text { CSA } \\
(\mu \mathrm{g} / \mathrm{mL})\end{array}$ & $\begin{array}{c}\text { LNPs } \\
(\mathrm{mg} / \mathrm{mL})\end{array}$ & $\begin{array}{c}\text { CSA } \\
(\mu \mathrm{g} / \mathrm{mL})\end{array}$ & $\begin{array}{c}\text { LNPs } \\
(\mathrm{mg} / \mathrm{mL})\end{array}$ \\
\hline $120 \%$ & 34.10 & 0.00 & 34.10 & 9.60 & 2.27 & 9.60 & 2.27 & 1.92 \\
\hline $100 \%$ & $28.42^{*}$ & $0.00^{*}$ & 28.42 & 9.60 & 1.89 & 9.60 & 1.89 & 1.92 \\
\hline $80 \%$ & 22.74 & 0.00 & 22.74 & 9.60 & 1.52 & 9.60 & 1.52 & 1.92 \\
\hline $60 \%$ & 17.05 & 0.00 & 17.05 & 9.60 & 1.14 & 9.60 & 1.14 & 1.92 \\
\hline $50 \%$ & 14.21 & 0.00 & 14.21 & 9.60 & 0.95 & 9.60 & 0.95 & 1.92 \\
\hline $30 \%$ & 8.53 & 0.00 & 8.53 & 9.60 & 0.57 & 9.60 & 0.57 & 1.92 \\
\hline $20 \%$ & 5.68 & 0.00 & 5.68 & 9.60 & 0.38 & 9.60 & 0.38 & 1.92 \\
\hline $0 \%$ & 0.00 & 0.00 & 0.00 & 9.60 & 0.00 & 9.60 & 0.00 & 1.92 \\
\hline
\end{tabular}

For the EDCD method, the data given in the table do not correspond to entrapped CSA because of the technical impossibility to spike drug inside nanoparticles. The value of the entrapped concentrations (from 0.00 to $2.27 \mu \mathrm{g} / \mathrm{mL}$ ) were hence calculated from the concentration of CSA spiked in ACN (from 0.00 to $3.41 \mu \mathrm{g} / \mathrm{mL}$ ), i.e. using a factor 1.5. For the TDCD method, the data were calculated as for the EDCD method. *: The $100 \%$ labelled sample of „CSA for FDCD“ was used as reference solution for the quantification of CSA by HPLC. The reference solution was prepared in duplicate. 


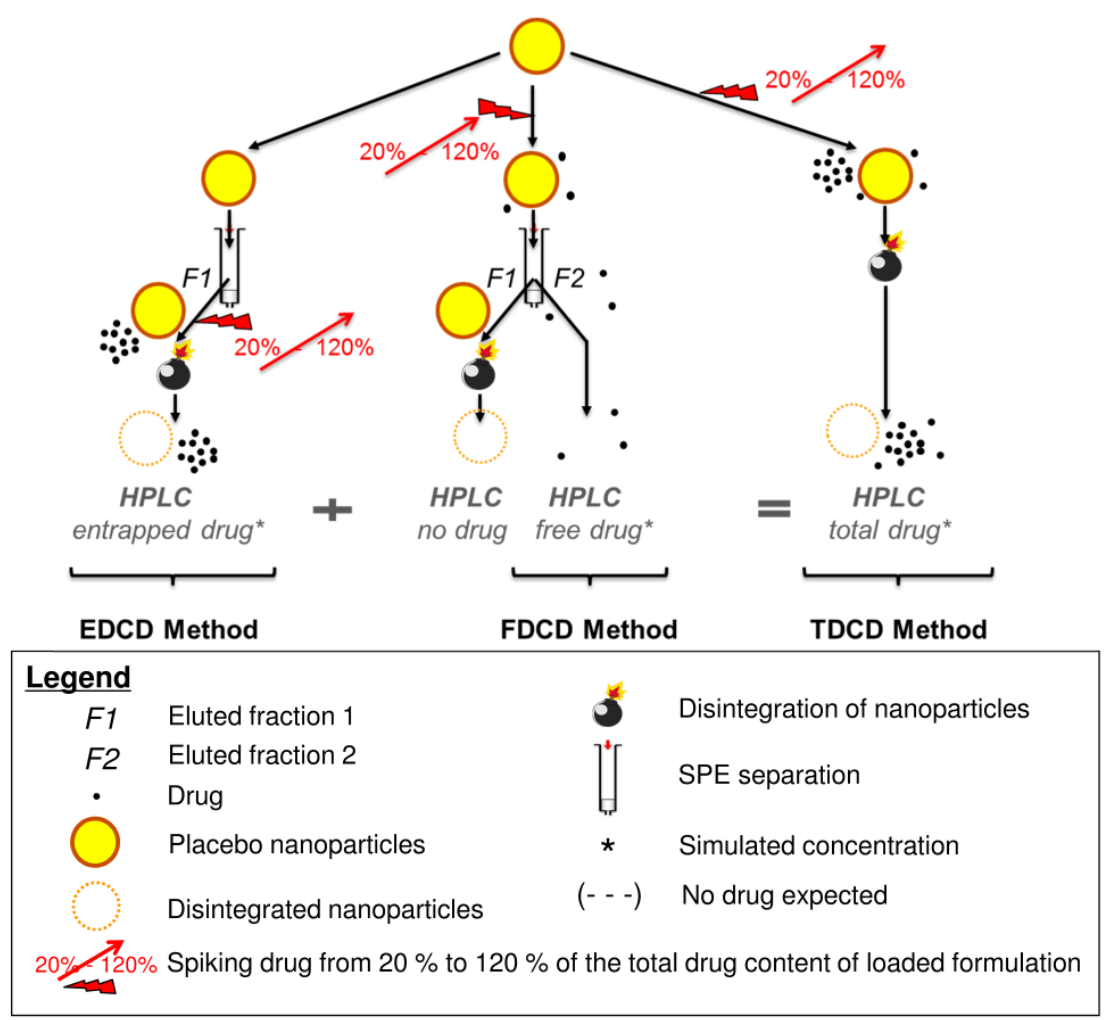

Figure 2: $\quad$ Validation plan for the tripartite SPE method.

\subsubsection{Validation FDCD method}

The FDCD method further consisted in maintaining the fractions F2 of eluted CSA in a water bath for 5 min at $37^{\circ} \mathrm{C}$. Aim of this step was to dissolve the precipitate formed by co-eluted excipients present in the extern phase of the Lipidot ${ }^{\circledR}$ formulation. The CSA was quantified by HPLC using the setups for FDCD (see in Table 3), compatible with the presence of co-eluted excipients. The specificity of the CSA separation was assessed from the fraction F1: the eluted LNPs were disintegrated as previously described and analyzed by HPLC to verify the absence of CSA in this fraction. The HPLC method used the setups for EDCD and TDCD (see in Table 3), compatible with the presence of excipients released after disintegration of the nanoparticles. 
Table 3: $\quad$ Setups of the HPLC methods used for FDCD, EDCD and TDCD

\begin{tabular}{|c|c|c|}
\hline Parameters & Setups for FDCD & Setups for EDCD and TDCD \\
\hline Column & \multicolumn{2}{|c|}{ XTerra ${ }^{\circledR}, \mathrm{RP}-18(5 \mu \mathrm{m} \times 150 \mathrm{~mm} \times 4.6 \mathrm{~mm})$} \\
\hline Elution mode & \multicolumn{2}{|c|}{ Isocratic } \\
\hline Mobile phase & $\mathrm{ACN}: \mathrm{H}_{3} \mathrm{PO}_{4} 5 \mathrm{mM}(75: 25, \mathrm{v} / \mathrm{v})$ & $\mathrm{MeOH}: \mathrm{H}_{3} \mathrm{PO}_{4} 5 \mathrm{mM}(75: 25, \mathrm{v} / \mathrm{v})$ \\
\hline Flow & $1.0 \mathrm{~mL} / \mathrm{min}$ & $1.2 \mathrm{~mL} / \mathrm{min}$ \\
\hline Wavelength detection & \multicolumn{2}{|c|}{$205 \mathrm{~nm}$} \\
\hline Temperature & \multicolumn{2}{|c|}{$55^{\circ} \mathrm{C}$} \\
\hline Injection volume & $30 \mu \mathrm{L}$ & $50 \mu \mathrm{L}$ \\
\hline Run time & $7 \mathrm{~min}$ & $10 \mathrm{~min}$ \\
\hline Retention time & $3.10 \min ( \pm 0.05 \mathrm{~min})$ & $6.55 \min ( \pm 0.10 \mathrm{~min})$ \\
\hline
\end{tabular}

\subsubsection{Validation EDCD method}

Concerning the EDCD method, the validation focused on the step following the elution of LNPs in F1, since spiking placebo Lipidot ${ }^{\circledR}$ with entrapped CSA was not feasible. Placebo LNPs were diluted using highly purified water until a concentration of $9.6 \mathrm{mg} / \mathrm{mL}$ LNPs (i.e. LNP dilution factor 6.25) and were further investigated in triplicate according to the overall SPE-plan presented in Table 1, until step 4, including the volume adjustment of $5 \mathrm{~mL}-\mathrm{VF} 1$ (i.e. LNP dilution factor 5). Afterward, $0.5 \mathrm{~mL}$ of $\mathrm{F} 1$ were mixed with $1 \mathrm{~mL}$ of a dilution set of CSA in acetonitrile containing $0.57,0.85,1.42,1.71,2.27,2.84$ and $3.41 \mu \mathrm{g} / \mathrm{mL}$ drug (dilution set designated by "CSA + placebo Lipidot ${ }^{\circledR}$ for EDCD" in Table 2). The resulting mixtures were shaken using the vortex to disintegrate the placebo LNPs and centrifuged for $10 \mathrm{~min}$ at $10,000 \mathrm{rpm}$ to precipitate the excipients and to only collect the CSA in supernatant solution. The HPLC setups used for EDCD are presented in Table 3.

\subsubsection{Validation TDCD method}

The TDCD method was validated by diluting the placebo Lipidot ${ }^{\circledR}$ using TFA until equivalent concentration of LNP to Fractions F1 in EDCD methods (1.92 mg/mL LNPs). As used for the validation of the EDCD method, $0.5 \mathrm{~mL}$ of this latter diluted solution were mixed afterward 
with $1 \mathrm{~mL}$ of the same dilution set of CSA in acetonitrile (dilution set designated by "CSA + placebo Lipidot ${ }^{\circledR}$ for TDCD" in Table 2). The mixtures were vortexed and centrifuged as previously described for the EDCD method and then analyzed using the same HPLC method (see Table 3).

\subsection{Implementation of the tripartite SPE method for a sample of cyclosporine A- loaded Lipidot ${ }^{\circledR}$ formulation and stress test}

The SPE protocol was implemented for a sample of cyclosporine A-loaded $120 \mathrm{~nm}$-sized Lipidot $^{\circledR}$ as previously validated. After equilibration at room temperature and dilution of the sample (dilution factor 6.25 in highly purified water), the FDCD and the EDCD methods were implemented directly whereas for the TDCD method, a dilution in TFA with factor 1:5 was applied. All samples were prepared five times $(n=5)$.

The protocol was implemented as well for the nanoformulation without the dilution step in highly purified water, namely $1 \mathrm{~mL}$ of Lipidot ${ }^{\circledR}$ product was placed in the SPE cartridge at step 3 (see Table 1) for the FDCD and EDCD methods and the product was directly diluted in TFA (dilution factor 1:5) for the TDCD method.

Regarding the stress conditions, $7 \mathrm{~mL}$ of cyclosporine A-loaded Lipidot ${ }^{\circledR}$ formulation were placed in the acceptor compartment of the vertical diffusion cell used without membrane as 1-compartment cell. The stress test was carried out for $48 \mathrm{~h}$ at $37^{\circ} \mathrm{C}$ with a magnetic stirring of $500 \mathrm{rpm}$. The stressed product was analyzed as previously without the dilution step in highly purified water. 


\section{3.}

RESULTS AND DISCUSSION

The developed protocol is based on the reverse-phase SPE concept, relying on the Van der Waals interactions between a hydrophobic analyte and a hydrophobic solid stationary phase (SPE-cartridge). This phenomenon leads to a stronger retention of the lipophilic analyte whereas the lipid nanoparticles are eluted faster by a hydrophilic mobile phase due to their outer hydrophilic PEG shell. The separation between the API and LNPs is further enhanced by the significant size difference of the two entities. Indeed, the stationary phase consists of beads containing pores smaller than the nanoparticle diameter but larger than the API as molecular entity. Based on these two mechanismes, PEG-shell lipid nanoparticles loaded with hydrophobic drugs or contrast agents can be eluted in a first step by using TFA as a hydrophilic mobile phase. In a second step, the retained drug can be eluted using ACN as a more lipophilic mobile phase. This technique is consequently suitable to separate and quantify either the remaining non-entrapped drug fraction after a given manufacturing process, to detect a leakage of drug during storage on the shelf, or even to monitor drug release during an in vitro performance test. Moreover, such separation of the API from the particles takes only few minutes whereas other techniques, like e.g. dialysis, may require several days, and thus might be of the same time scale as the expected drug release from the carrier. Contrary to polymer or inorganic nanoparticles, considering the metastable character of lipid nanoparticles when dispersed in aqueous buffer, techniques based on separation-precipitation cannot be applied. Methods requiring organic solvents would destabilize the interface of droplets resulting in a biphasic system (oil and water parts). Consequently, solid phase extraction turned out to be the most suitable separation method for lipid nanoparticles.

\subsection{Assessment of the colloidal stability of Lipidot ${ }^{\circledR}$ following SPE protocol}

As colloidal nanoemulsions, Lipidot ${ }^{\circledR}$ formulations are composed of lipid droplets surrounded by lecithin and coated with PEG surfactant with Z-average diameters from 50 to $150 \mathrm{~nm}$ to render colloidal stability, reported with a shelf-life in suspension over one year(12). Because 
their interaction with the stationary phase materials could potentially affect the integrity of the nanoparticles it was necessary to confirm their colloidal stability after the SPE process to validate its use as a separation method. Such experiment was consequently performed using a Zetasizer Nano ZS after SPE process. It was firstly shown that the median size and Polydispersity Index (PdI) for different dilutions of LNPs in TFA (control dilutions) did not significantly differ one to another. This finding demonstrated that acid medium did not destabilize the LNPs. Secondly, obvious differences between the particle size of the LNPs eluted in TFA after SPE and the control-LNPs diluted in TFA were observed for the concentrations $3 \mathrm{mg} / \mathrm{mL}$ LNPs (no peak observed after SPE) and $6 \mathrm{mg} / \mathrm{mL}$ LNPs (delayed peak corresponding to larger particle sizes after SPE) (Figure 3 and Table 4). This suggests that nanoparticle concentration of $6 \mathrm{mg} / \mathrm{mL}$ and below should not be used.

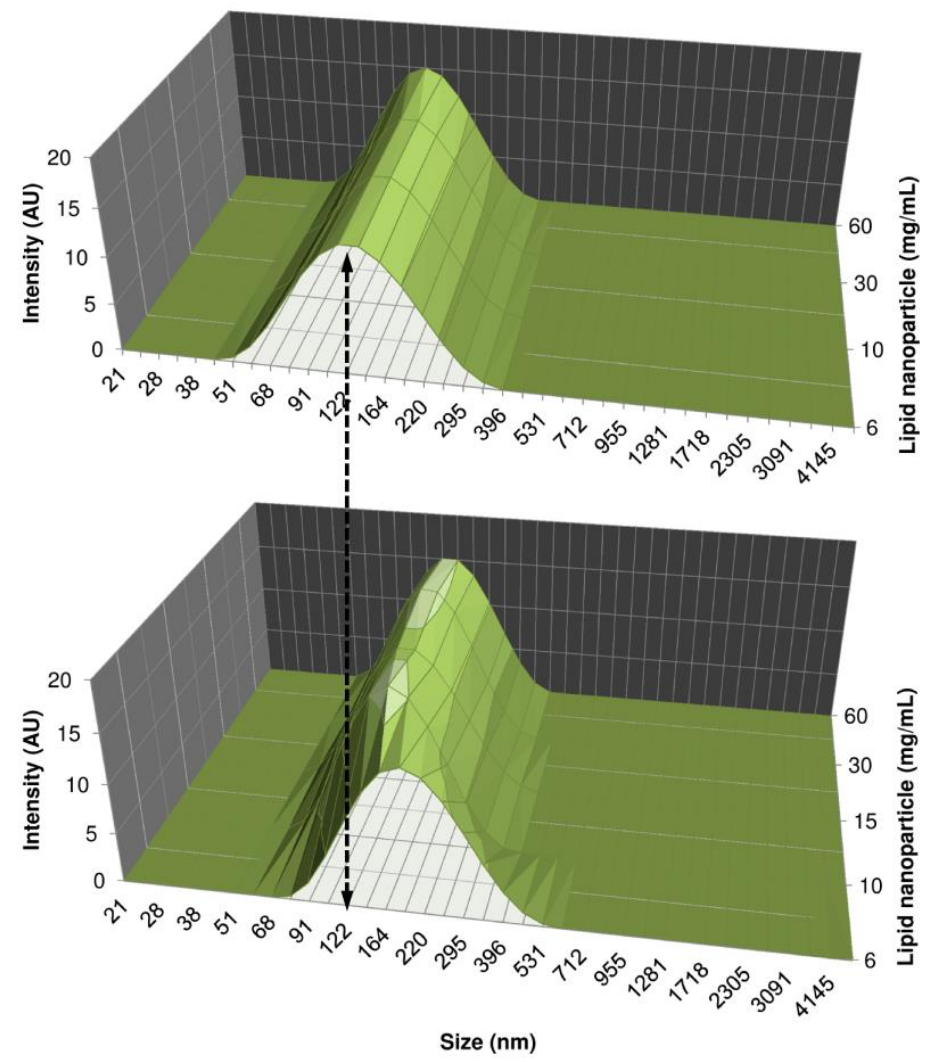

Figure 3: $\quad$ Particle distribution for different concentrations of Lipidot $^{\circledR}$ samples before and after SPE. The control set of LNPs (top) was diluted until same concentration as the set of eluted LNPs through SPE (bottom). The size distribution remained constant for the whole control set $(6,10,30$ and $60 \mathrm{mg} / \mathrm{mL})$. After SPE, the modal size remained not affected for the concentrations higher than $10 \mathrm{mg} / \mathrm{mL}$ but was shifted towards higher values at $6 \mathrm{mg} / \mathrm{mL}$. 
Table 4: Z-average diameter $(\mathrm{nm})$ and polydispersity index (Pdl) for different concentrations of Lipidot ${ }^{\circledR}$ samples prepared by SPE or not (Control).

\begin{tabular}{ccccc}
\hline & \multicolumn{2}{c}{ Z-average diameter $(\mathbf{n m})$} & \multicolumn{2}{c}{ Pdl } \\
\hline LNP concentration $(\mathbf{m g} / \mathbf{m L})$ & Control & SPE & Control & SPE \\
\hline 3 & n. a. & 2849.0 & n. a. & 0.747 \\
6 & 123.3 & 169.3 & 0.125 & 0.129 \\
10 & 124.8 & 137.0 & 0.117 & 0.097 \\
15 & n. a. & 130.9 & n. a. & 0.141 \\
30 & 124.1 & 125.8 & 0.114 & 0.106 \\
60 & 124.4 & 123.8 & 0.125 & 0.106 \\
\hline
\end{tabular}

\subsection{Validation of the tripartite SPE method}

The tripartite SPE method actually consists of preparative and analytical methods for the quantification of the total API (TDCD), the entrapped API (EDCD) and the free API (FDCD). The dilution set of CSA prepared without LNPs for the validation of the FDCD method was practically not turbid in the selected cyclosporine A concentration range. However, for concentrations greater than $35-40 \mu \mathrm{g} / \mathrm{mL}$ (before SPE), the turbidity increased, precipitates were observed and the validation test failed (RSD $>5 \%$ ). The validation of FDCD succeeded using highly purified water as dilution medium until $34.10 \mu \mathrm{g} / \mathrm{mL}$ (see results in Table 5). The linearity and repeatability were slightly better in presence than in absence of Lipidot $^{\circledR}$, obviously reflecting the improved solubility of cyclosporine $A$ by this formulation. 


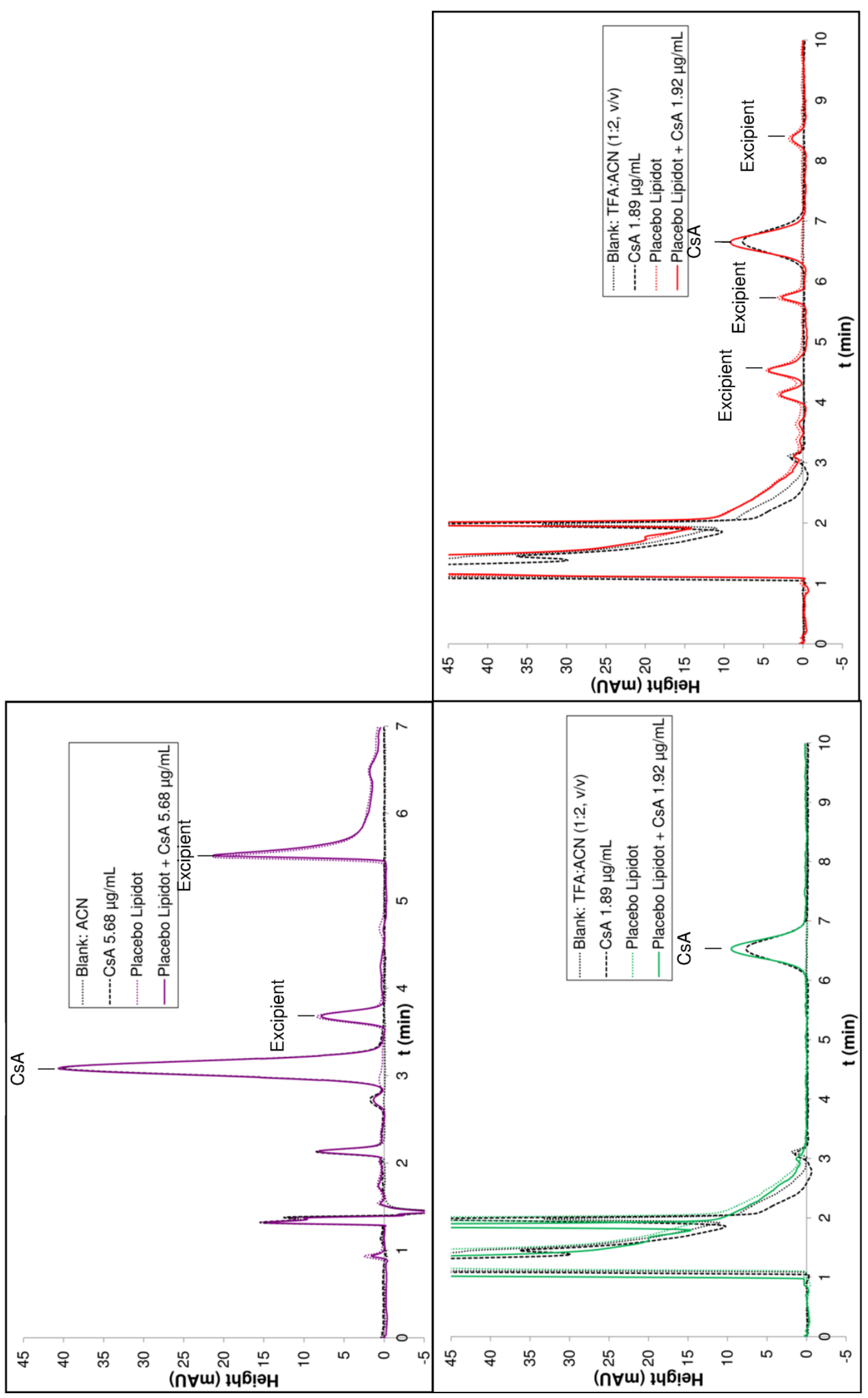

Figure 4: Chromatogram of cyclosporine A quantified using the FDCD method (violet), EDCD method (green) and TDCD method (red) 


\subsection{Tripartite SPE method for a Lipidot ${ }^{\circledR}$ nanoformulation - Stress test}

A protocol of characterization based on the SPE technique was previously designed, developed and validated using placebo lipid nanoparticles. Its implementation was also performed using the loaded Lipidot $^{\circledR}$ formulation. The tripartite SPE method was able to determine the concentrations of entrapped CSA $(88.9 \mu \mathrm{g} / \mathrm{mL})$, free CSA $(67.5 \mu \mathrm{g} / \mathrm{mL})$ and total CSA $(163.8 \mu \mathrm{g} / \mathrm{mL})$. Moreover, comparing the experimental total CSA concentration to the theoretical concentration $(210.0 \mu \mathrm{g} / \mathrm{mL})$, an encapsulation efficiency of the manufacturing process could be calculated (78\%) to highlight the loss of non-encapsulated drug removed over intensive dialysis during the manufacturing process. The protocol was further implemented using a diluted formulation of Lipidot ${ }^{\circledR}$ in order to assess the influence of a dilution on the release of CSA. The free CSA represented $41 \%$ of the total concentration for the samples prepared without dilution, vs. $82 \%$ for the diluted samples. The entrapped CSA was quantified as well and represented $54 \%$ for undiluted sample vs. $11 \%$ for diluted samples. The methods for free and entrapped CSA could successfully reveal a dilution-effect and were almost complementary. However, according to the results summarized in Table 6, the repeatabilities (coefficient of variation) were very satisfying for the total and free CSA content determination method but not optimal for the entrapped CSA. Consequently, when the complementarity between the three methods was not completely achieved, the unidentified amount should be to be related to the entrapped CSA. As concern the results of the stress test at $37^{\circ} \mathrm{C}$ and $500 \mathrm{rpm}$, a proportion of $68 \%$ free CSA was observed after the stress test vs. $41 \%$ free CSA without the test. The result suggested a sustained release potential for the Lipidot ${ }^{\circledR}$ formulations. All the results of the tests performed for CSA-loaded Lipidot $^{\circledR}$ formulation are given in Table 6. 


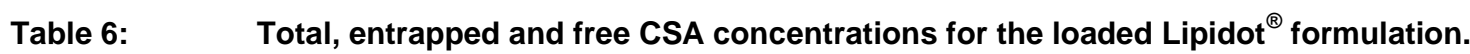

\begin{tabular}{|c|c|c|c|c|c|c|}
\hline & \multicolumn{2}{|c|}{ No Dilution } & \multicolumn{2}{|c|}{ Dilution 6.25} & \multicolumn{2}{|c|}{$\begin{array}{l}\text { No Dilution } \\
\text { Stress test }\end{array}$} \\
\hline & $\begin{array}{c}\text { Mean } \\
(\mu \mathrm{g} / \mathrm{mL})\end{array}$ & $\begin{array}{l}\text { CV } \\
(\%)\end{array}$ & $\begin{array}{c}\text { Mean } \\
(\mu \mathrm{g} / \mathrm{mL})\end{array}$ & $\begin{array}{l}\text { CV } \\
(\%)\end{array}$ & $\begin{array}{c}\text { Mean } \\
(\mu \mathrm{g} / \mathrm{mL})\end{array}$ & $\begin{array}{l}\text { CV } \\
(\%)\end{array}$ \\
\hline Total CSA & $\begin{array}{r}163.8 \\
100\end{array}$ & 1.1 & $\begin{array}{r}155.5 \\
100\end{array}$ & 1.1 & $\begin{array}{r}178.8 \\
100\end{array}$ & 1.1 \\
\hline $\begin{array}{l}\text { Entrapped CSA } \\
\qquad \% / \text { Total CSA }\end{array}$ & \begin{tabular}{ll|}
88.9 & \\
& 54
\end{tabular} & 8.5 & $\begin{array}{r}17.6^{\star \star} \\
11\end{array}$ & $15.3^{\star *}$ & $\begin{array}{l}39.0 \\
\\
\end{array}$ & 15.3 \\
\hline$\% /$ Total CSA & $\begin{array}{ll}67.5^{*} & \\
& 41\end{array}$ & $6.3^{*}$ & $\begin{array}{r}127.1 \\
82\end{array}$ & 5.0 & $\begin{array}{r}121.5 \\
68\end{array}$ & 4.0 \\
\hline $\begin{array}{r}\text { Total - (Entrapped + Free) } \\
\% / \text { Total CSA }\end{array}$ & 7.4 & n.a. & 10.8 & n.a. & $\begin{array}{l}18.3 \\
10\end{array}$ & n.a. \\
\hline
\end{tabular}

Dilution 1:1: sample not diluted prior SPE separation; Dilution 6.25: sample diluted prior SPE separation; Dilution 1:1 Stress test: sample stressed over $48 \mathrm{~h}$, not diluted prior SPE. Experiments performed for $n=5 .{ }^{*}: n=4 .{ }^{* *}: n=3$. CV: coefficient of variation. n.a.: not applicable. 


\section{CONCLUSION}

An accurate, repeatable, fast and automatable method based on SPE was developed for the separation and quantification of non-entrapped and entrapped cyclosporine $A$ in the lipid nanoformulation Lipidot ${ }^{\circledR}$. The results obtained by DLS have shown that nanoparticles were actually eluted in a first fraction. Furthermore, the validation of the technique by HPLC resulted in satisfying results revealing a reliable analytical performance of the method and usability for quality control purposes. At this stage of product developement, the SPE technique clearly demonstrated the complexity of the Lipidot ${ }^{\circledR}$ formulation and underlined the need of further characterization investigations. Indeed, in vitro drug release tests remain necessary to confirm the sustained release potential of Lipidot ${ }^{\circledR}$ and to elucidate the mechanism of release (e.g. diffusion, erosion, melting, etc.). Further improvements towards a pharmaceutical product are required as well. Besides testing drug release and leakage during storage, respectively, the technique would also allow to quantify the amount of API removed during the purification step of the manufacturing process and thus the encapsulation rate and efficacy. Apart from the considerations related to the specific formulation, which served as an example for the study, the concept of SPE presents itself as promising tool for drug development and quality control purposes of lipid-based nanopharmaceuticals in an industrial environment. 


\section{ACKNOWLEDGEMENTS}

This work was sponsored by the German Federal Ministry of Education and Research and financially supported by the EuroNanoMed Project Delivering Nanopharmaceuticals through Biological Barriers 'BiBa' (ERA-Net EuroNanoMed Project Number 13N11846).

The authors thank Christina Draheim for performing the DLS preliminary tests and Johannes Krämer for providing the facility and equipment necessary for the investigations. 


\section{BIBLIOGRAPHY}

1. Venditto VJ, Szoka FC, Jr. Cancer nanomedicines: so many papers and so few drugs! Advanced drug delivery reviews. 2013;65(1):80-8.

2. Crist RM, Grossman JH, Patri AK, Stern ST, Dobrovolskaia MA, Adiseshaiah PP, et al. Common pitfalls in nanotechnology: lessons learned from NCl's Nanotechnology Characterization Laboratory. Integrative biology : quantitative biosciences from nano to macro. $2013 ; 5(1): 66-73$.

3. Shah RB, Khan MA. Nanopharmaceuticals: challenges and regulatory perspective. In: De Villiers MM, Aramwit P, Kwon GS, editors. Nanotechnology in Drug Delivery2009. p. 627.

4. Modi S, Anderson BD. Determination of drug release kinetics from nanoparticles: overcoming pitfalls of the dynamic dialysis method. Molecular pharmaceutics. 2013;10(8):3076-89.

5. Lopez-Serrano A, Olivas RM, Landaluze JS, Camara C. Nanoparticles: a global vision. Characterization, separation, and quantification methods. Potential environmental and health impact. Analytical Methods. 2014;6(1):38-56.

6. Magenheim B, Levy MY, Benita S. A new in vitro technique for the evaluation of drug release profile from colloidal carriers - ultrafiltration technique at low pressure. International Journal of Pharmaceutics. 1993;94(1-3):115-23.

7. Wallace SJ, Li J, Nation RL, Boyd BJ. Drug release from nanomedicines: Selection of appropriate encapsulation and release methodology. Drug delivery and translational research. 2012;2(4):284-92.

8. Ohnishi N, Yamamoto E, Tomida H, Hyodo K, Ishihara H, Kikuchi H, et al. Rapid determination of the encapsulation efficiency of a liposome formulation using columnswitching HPLC. International Journal of Pharmaceutics. 2013;441(1-2):67-74. 
9. Griese N, Blaschke G, Boos J, Hempel G. Determination of free and liposomeassociated daunorubicin and daunorubicinol in plasma by capillary electrophoresis. Journal of chromatography A. 2002;979(1-2):379-88.

10. Bibette J, Goutayer M, Texier-Nogues I, inventors; Commissariat à I'Energie Atomique, assignee. Method for preparing nano-emulsions patent WO2008104717 (A2). 2008.

11. U. S. Food and Drug Administration. Guideline for Industry: Text on Validation of Analytical Procedures : ICH-Q2A: U.S. Department of Health and Human Services, Public Health Service, Food and Drug Administration; 1995.

12. Delmas T, Piraux H, Couffin A-C, Texier I, Vinet F, Poulin P, et al. How to prepare and stabilize very small nanoemulsions. Langmuir. 2011;27(5):1683-92.

13. Delmas T, Fraichard A, Bayle P-A, Texier I, Bardet M, Baudry J, et al. Encapsulation and release behavior from lipid nanoparticles: model study with Nile red fluorophore. Journal of Colloid Science and Biotechnology. 2012;1(1):16-25. 